REVISTA DE GESTÃO E SECRETARIADO

MANAGEMENT AND ADMINISTRATIVE

PROFESSIONAL REVIEW

ISSN: 2178-9010
Revista GeSec

São Paulo, SP, Brasil

v. 12 , n. 2, p. $122-146$

maio /ago. 2021

DOI: http://dx.doi.org/10.7769/gesec.v12i2.1177

\title{
Gestão de eventos: um estudo bibliométrico e sociométrico da produção científica internacional
}

\section{Event management: a bibliometric and sociometric study of international scientific production}

\author{
Francisco Edson Rodrigues da Silva ${ }^{1}$ \\ Joelma Soares da Silva ${ }^{2}$ \\ Elaine Freitas de Sousa ${ }^{3}$ \\ Antonio Roberth Moreira de Alencar Costa ${ }^{4}$
}

\section{Resumo}

O objetivo deste artigo é apresentar as características da produção científica internacional sobre gestão de eventos. Foram analisadas características de uma amostra de 338 artigos disponibilizados na base Scopus, de 1991 a 2019, no que tange aos aspectos de autoria, periódicos, trabalhos mais citados, temáticas centrais, redes de coautoria, de cocitação, e de acoplamento bibliográfico. O trabalho justifica-se na necessidade do entendimento e da sustentação de uma identidade de pesquisa sobre eventos apontada por Baum, LockstoneBinney e Robertson (2013). Trata-se de uma pesquisa de natureza quantitativa, descritiva, construída por meio de estudo bibliométrico e sociométrico. Os dados foram analisados com o auxílio dos softwares Microsoft Excel e VOSviewer. Após análise dos dados, foi possível inferir que a pesquisa na área de gestão de eventos encontra-se em ascensão, concentrando-se principalmente na Austrália, Estados Unidos e Reino Unido - países que apresentam cursos de formação de nível superior específicos para o campo de gestão de eventos. Identificou-se uma lacuna de produção brasileira em bases internacionais. Ainda, as temáticas de pesquisa em evidência são educação, turismo, marketing, sustentabilidade e eventos verdes.

Palavras-chave: Gestão de Eventos. Bibliometria. Sociometria.

\footnotetext{
${ }^{1}$ Graduado em Administração, Técnico-Administrativo em Educação da Universidade Federal do Ceará (UFC).

2 Doutora em Administração, Docente na UFC.

${ }^{3}$ Doutora em Educação, Docente da UFC.

${ }^{4}$ Graduando em Secretariado Executivo na UFC.
} 


\begin{abstract}
This research aims to present the characteristics of international scientific production about event management. The characteristics of a sample of 338 articles available at Scopus, from 1991 to 2019, were analyzed regarding authorship, journals, most cited researches, central topics and co-authoring, co-citation and bibliographic coupling networks. The research is based on the need to understand and sustain a research identity on events pointed out by Baum, Lockstone-Binney e Robertson (2013). It is a quantitative, descriptive research, built through bibliometric and sociometric study. For the data analysis, Microsoft Excel and VOSviewer softwares were used. After analyzing the data, it was possible to infer that the research about event management is on the rise, focusing mainly on Australia, the United States and the United Kingdom - countries that have specific higher education courses for the field of event management. A gap of Brazilian production was identified on international bases. Also, thematic trends are education, tourism, marketing, sustainability and green events.
\end{abstract}

Keywords: Event management. Bibliometrics. Sociometrics.

\title{
Introdução
}

A vida humana é pautada por eventos. Desde uma gestação, enquanto ato social, a existência de um sujeito é circundada por eventos. Ao longo da história, os eventos foram profissionalizados e, consequentemente, segmentados em diferentes tipologias, classificações, objetivos, entre outros aspectos. Assim, sendo reconhecidos enquanto importante segmento social e econômico de uma sociedade (Bowdin, Allen, O’Toole, Harris \& McDonnell, 2011; Getz, 2012), os eventos passaram a demandar formas de gestão próprias que atendessem as suas características específicas (Wagen \& Carlos, 2005).

A literatura científica não se manteve à parte desse olhar diferenciado sobre os eventos, embora ainda careça de aprofundamentos. Para Baum, Lockstone-Binney e Robertson (2013), embora valiosas revisões já tenham sido realizadas para identificação do escopo dos estudos sobre eventos e as lacunas do conhecimento atual, ainda não se lançou, até o momento, um olhar crítico sobre o campo, a fim de avaliar seu desempenho acadêmico, posição e mérito. $\mathrm{Na}$ esteira do pensamento dos autores, a produção científica brasileira sobre eventos também carece desse olhar crítico ou mesmo de um debate teórico sobre o tema.

Nesse sentido, este estudo parte da seguinte questão: como se caracteriza a produção científica internacional sobre gestão de eventos? O objetivo geral é apresentar as características 
da produção científica internacional sobre gestão de eventos, especificamente no que tange aos aspectos de autoria, periódicos, trabalhos mais citados, temáticas centrais, redes de coautoria, de cocitação e de acoplamento bibliográfico.

Buscando atingir o objetivo, foi realizado um estudo bibliométrico e sociométrico na Scopus, considerada a maior base de dados multidisciplinar da literatura científica no mundo, abrigando pesquisas de qualidade e de alto fator de impacto (Durán-Sánchez, Río-Rama \& Álvarez-García, 2016; Baas, Schotten, Plume, Côté \& Karim, 2020), o que justifica a sua escolha para o trabalho.

Dessa forma, este estudo avança e explicita características da evolução e da realidade atual da produção científica internacional sobre gestão de eventos e das lacunas de abordagens não contempladas, conforme recomendam Teixeira, Iwamoto e Medeiros (2013). Assim, espera-se contribuir com a geração de novos conhecimentos, sob enfoques de olhares descolonizados (Teixeira, Iwamoto \& Medeiros, 2013).

O estudo está dividido em seis seções, incluindo esta introdução. Apontamos o referencial teórico sob os eixos temáticos gestão de eventos e estudos bibliométricos e sociométricos. Na seção três são apresentados os procedimentos metodológicos, seguidos dos resultados e discussões. Por fim, apresentam-se as considerações finais e as referências que subsidiaram o estudo.

\section{Referencial Teórico}

\subsection{Gestão de eventos}

A Gestão de Eventos é uma temática peculiar com relação aos estudos e pesquisas sobre a área de gestão, devido às suas particularidades quanto aos aspectos de temporalidade, geográficos e sazonais, que devem ser levados em consideração quando se atua nas funções de planejamento, organização, direção e controle (Wagen \& Carlos, 2005).

Esta pesquisa adota o conceito de Getz (2007) para evento, que o define como um acontecimento que se dá em local e tempo definidos, com começo e fim determinados, envolvendo um conjunto especial de circunstâncias. A esse conceito soma-se o de Zanella (2011), para quem evento pode ser definido como uma concentração de pessoas, em prol do objetivo de celebrar acontecimentos e/ou datas comemorativas, em locais, datas e horários préestabelecidos.

Em complemento, Getz (2007) aponta os aspectos subjetivos que devem ser considerados quando se trata de gerir eventos, o que envolve as motivações, necessidades, 
preferências, emoções e percepções de gestores e do público. Esses aspectos subjetivos compõem as experiências e os significados dos eventos (Getz, 2007).

Nesse contexto, a gestão de eventos leva em conta fatores internos, como recursos, planejamento e processos gerenciais (Silvers, Bowdin, O’Toole \& Nelson, 2006) e externos, que são determinantes para a execução dos eventos. Tais fatores externos podem ser de ordem social, econômica, cultural e política (Getz, 2007; Zanella, 2011). Assim, esses fatores segmentam os eventos, destacando-os por sua natureza específica.

Dessa forma, de acordo com Zanella (2011), os eventos podem ser classificados em diversas categorias, tais como comerciais, culturais, sociais, artístico/culturais, gastronômicos, esportivos, políticos, históricos, religiosos, científicos ou técnicos, ressaltando que, para cada categoria, custos, programas, profissionais qualificados e ações distintas são necessárias para que cada evento seja único (Silvers et al., 2006).

Percebe-se, portanto, que no campo prático e de mercado de trabalho, muitas são as possibilidades de atuação na gestão de eventos, se caracterizando como uma área multidisciplinar que demanda diferentes habilidades e talentos, envolvendo processos como marketing, gestão de risco, logística e comunicação (Silvers et al., 2006).

Já enquanto campo de estudo, a gestão de eventos é um tópico recente e que tem abrangido diferentes abordagens, conforme apontam Park e Park (2017). Os autores demonstram que as pesquisas sobre gestão de eventos agrupam-se em três temáticas principais (marketing, gestão e turismo), que abordam assuntos como planejamento, avaliação, formação e tecnologia (Park \& Park, 2017). Além disso, Park e Park (2017) reforçam o marketing como a temática de gestão de eventos que mais cresceu nas pesquisas, e destacam as pesquisas relacionadas ao impacto econômico e social dos eventos nas comunidades em que acontecem, bem como a avaliação de satisfação dos participantes e sua intenção de repetir a experiência.

Em um esforço anterior, Mair e Whitford (2013) identificaram que a produção científica sobre gestão de eventos apontava para temáticas como o impacto e o legado dos eventos, políticas e governança, e a relação entre eventos e cultura/identidade. O estudo de Mair e Whitford (2013) concluiu que eram necessários mais estudos relacionando eventos e seus impactos socioculturais e ambientais.

Assim, na perspectiva de Baum et al. (2013), os estudos sobre eventos desdobram-se nos campos do turismo, lazer e hospitalidade, mas está para além deles. Ocorre, porém, que segundo os próprios autores, existem poucos periódicos que atendem ao campo de estudo dos eventos e, muitos, tinham menos de cinco anos à época da pesquisa dos autores. Desta forma, os pesquisadores de estudos de eventos estão publicando amplamente fora do campo em busca 
de espaços conceituados no turismo, lazer e hospitalidade, movimento esse outrora realizado por pesquisadores destes campos de estudo. Por fim, os autores reforçam o pensamento de Getz (2012) acerca da necessidade de uma lente mais teórica para os estudos de eventos.

\subsection{Bibliometria e sociometria}

O estudo bibliométrico centra-se na investigação que tem como objeto de estudo a quantificação da produção científica. Assim, a bibliometria é uma forma de tentar quantificar os processos de comunicação escrita (Alvarenga, 1998).

De maneira geral, adotam-se três indicadores bibliométricos para análise de produção científica: a lei de Lotka, a lei de Bradford e a lei de Zipf (Machado, Souza, Parisotto, \& Palmisano, 2016). A lei de Lotka (1926) propõe que um pequeno número de pesquisadores é mais profícuo em determinada área do conhecimento, produzindo um volume considerável de estudos, enquanto muitos pesquisadores produzem pouco na mesma área.

A lei de Bradford, também conhecida como lei da Dispersão (Rousseau \& Rousseau, 2000), diz respeito aos periódicos que mais publicam em determinado tema. Assim como em Lotka, a lei de Bradford propõe que um núcleo pequeno de periódicos concentra a maior parte de publicações sobre um assunto, enquanto há uma região, ao redor desse núcleo, composta por muitos periódicos com pouca produtividade nesse mesmo assunto.

Por fim, a lei de Zipf ou do Mínimo Esforço (Chang, 2016) mede a frequência com que determinado termo (palavra-chave, por exemplo) aparece um texto, podendo indicar sua temática central (Lima \& Leocádio, 2018).

Por sua vez, a sociometria consiste na exploração, mapeamento e mensuração das relações constituídas entre forças sociais individuais que atuam em redes de interação (Moreno, 1992). A análise sociométrica auxilia na identificação da relação entre autores e instituições, e como essa interação constitui o conhecimento em determinada área (Rosa, Mendes, Teixeira, $\&$ Martins, 2010). Nesse sentido, analisam-se os atores ou nós, isoladamente, bem como suas relações; os laços ou elos; as reciprocidades e o tamanho das redes. Exemplos de análise sociométricas são as redes de coautoria, de cocitação e de acoplamento bibliográfico.

As redes de coautoria se formam quando pesquisadores compartilham a autoria de trabalhos científicos. Analisar essas redes identifica como autores, instituições e países se relacionam no que tange aos estudos que realizam e publicam em conjunto (Rosa et al., 2010; Eck \& Waltman, 2014). 
A análise de cocitação, por sua vez, possibilita identificar documentos ou autores que são frequentemente citados juntos. Isso permite inferir agrupamentos de conteúdo, uma vez que quanto maior for essa frequência, mais proximidade de conteúdos esses fatores devem possuir (Cardoso, Martins \& Kato, 2015).

Por fim, a análise de acoplamento bibliográfico mede a similaridade das fontes de documentos, utilizando-se da contagem do número de referências bibliográficas que são compartilhadas por eles (Cardoso et al., 2015). Assim, quanto mais próximos dois autores se encontrarem na rede, maior é o acoplamento entre eles, o que pode indicar núcleos referenciais de pesquisas (Lima \& Leocádio, 2018).

Dessa forma, estudos bibliométricos e sociométricos colaboram com a descrição de um campo do conhecimento, contribuindo com a ampliação de uma área de estudo (Sousa, Fontenele, Silva, \& Sousa, 2019; Zago, Domingues \& Silva, 2019). Para além de auxiliar na construção de um panorama sobre determinada temática, possibilitam também apontar lacunas, tendências e oportunidades de pesquisa.

\section{Metodologia}

Partindo de uma abordagem quantitativa, esta pesquisa constituiu-se a partir de uma metodologia bibliométrica e sociométrica para analisar aspectos de autoria, periódicos, temáticas mais abordadas, trabalhos mais citados, redes de coautoria, de cocitação e de acoplamento bibliográfico na produção científica internacional sobre gestão de eventos.

A pesquisa tomou como base de trabalhos científicos a plataforma Scopus, reconhecida internacionalmente pela comunidade científica por apresentar uma grande quantidade de obras de qualidade e alto impacto.

A amostra analisada foi constituída a partir de três fases. Na primeira, realizou-se consulta na base Scopus pelo descritor "event* management" (uso de asterisco para retornar resultados tanto no singular, quanto no plural; e entre aspas para retornar resultados que continham o termo exato), buscando no campo Títulos, Resumos e Palavras-chave, sem qualquer restrição de período. Para essa primeira etapa, foram encontrados 1.857 documentos. Na segunda fase, restringiu-se o tipo de documento e o idioma da publicação, optando-se apenas por artigos em língua inglesa, reduzindo a amostra inicial para 873 artigos. Por fim, realizou-se uma leitura sistemática dos resumos dos 873 artigos, a fim de identificar quais se adequavam à temática de estudo, resultando em uma amostra final de 338 artigos. 
Após a constituição da amostra de artigos, a análise dividiu-se em duas etapas. $\mathrm{Na}$ primeira, com utilização do software Microsoft Excel, foram analisados a evolução temporal da produção, as características de autoria, os autores e periódicos mais profícuos e os trabalhos mais citados. Na segunda etapa, com utilização do software VOSviewer, foram analisadas a redes de coocorrência de palavras-chave, de coautoria, de cocitação e de acoplamento bibliográfico.

\section{Análise e Discussão dos Resultados}

\subsection{Evolução temporal da produção}

Conforme a Figura 1, as publicações sobre gestão de eventos têm crescido nos últimos anos. Antes dos anos 2000, representavam uma produção ínfima, variando seus objetos de estudo entre grandes eventos e educação em turismo. A partir dos anos 2000, as publicações começam a crescer, principalmente no final da década, confirmando tendência apontada por Park e Park (2017).

Do ano de 2008 para 2009, por exemplo, as publicações quase triplicaram. Os trabalhos desses anos mostram uma área de estudos mais consolidada, com ênfase em eventos de grande porte (como olimpíadas e campeonatos esportivos) e abrangendo discussões como epistemologia da área, profissionalização do campo, a figura do gestor de eventos, relações com stakeholders, e um início de discussão sobre o impacto dos eventos nas comunidades em que ocorrem e sustentabilidade.

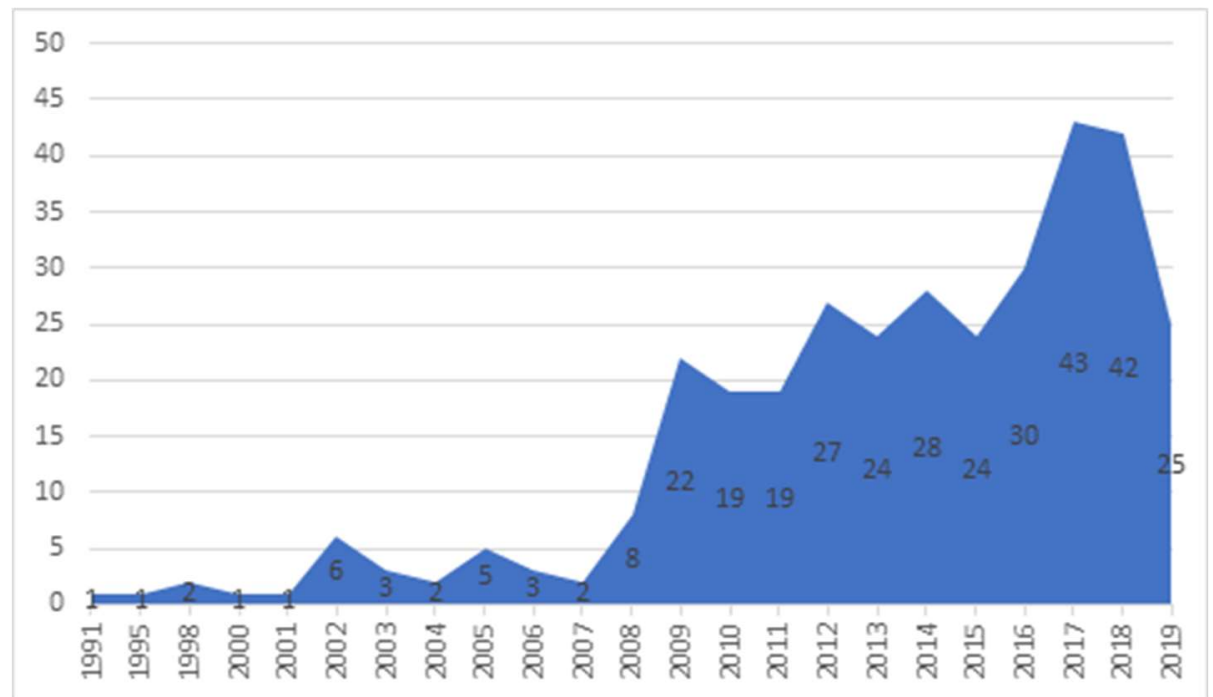

Figura 1. Evolução temporal

Fonte: elaborado pelos autores (2019). 
A produção continuou a crescer nos anos seguintes, com pequenas variações entre 2010 e 2015, e atingiu seu ápice em 2017, com 43 publicações. Nesse cenário, destacaram-se trabalhos com temáticas sobre eventos verdes e sustentáveis, o que sinaliza uma preocupação da área em alinhar suas práticas com a pauta da sustentabilidade ambiental.

Em relação a 2019, até a data de coleta de dados desta pesquisa, foram localizados 25 trabalhos com temas diversos, desde sustentabilidade à identidade acadêmica. De maneira geral, a evolução temporal da pesquisa em gestão de eventos tem demonstrado o crescimento do interesse pela área, que parece dialogar com temáticas que vão além da gestão, incluindo sustentabilidade e profisssionalização.

\subsection{Características de autoria}

Os 338 artigos que compõem a amostra desta pesquisa são de autoria de 383 autores. A seguir são expostos e discutidos os autores, instituições e países que mais publicam, bem como a composição dessa autoria.

\subsubsection{Autores que mais publicam}

A partir dos 383 autores identificados, buscou-se analisar aqueles que mais publicam na área de gestão de eventos. Para fins de visualização, a Figura 2 mostra apenas aqueles que tiveram 6 ou mais artigos publicados no período abrangido pela pesquisa.

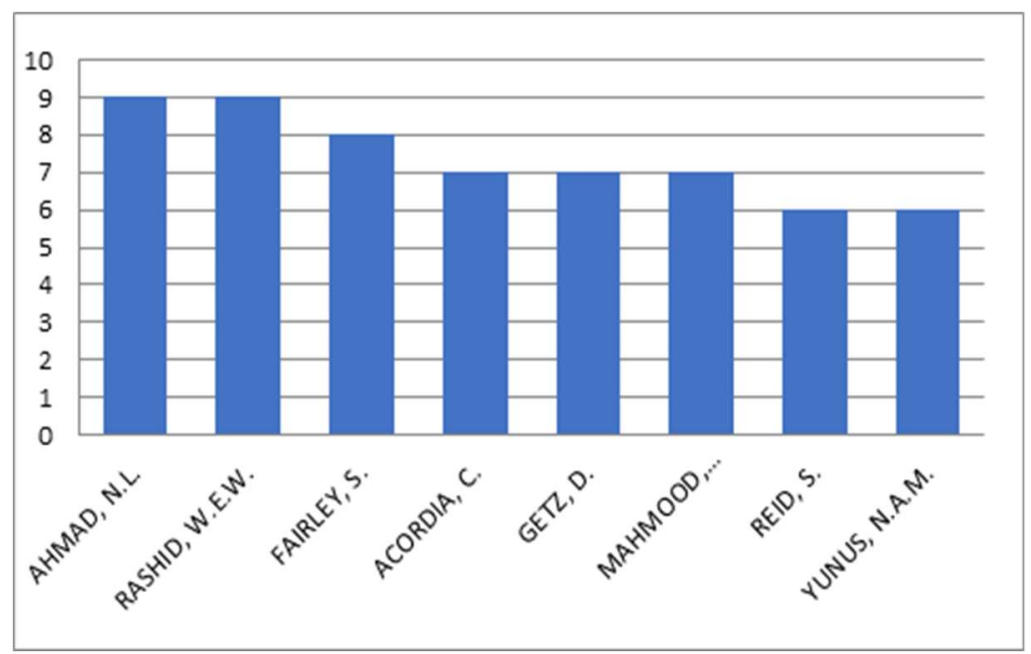

Figura 2. Autores que mais publicam

Fonte: elaborado pelos autores (2019). 
Os oito autores destacados na Figura 2 (que representam 2\% do total de autores de toda a amostra) é responsável por 33 dos 338 artigos (aproximadamente 10\%). A esses dados, acresce-se o fato de que os outros 375 autores publicaram, entre 1991 e 2019, o máximo de quatro artigos (289 publicaram apenas um). Esse cenário demonstra uma produção dispersa e confirma a lei bibliométrica de Lotka, segundo a qual um pequeno grupo de autores é responsável pelo maior número de publicações sobre um tema, e muitos autores publicam pouco, conforme apontam Machado et al. (2016).

Assim, conforme a Figura 2, os autores que mais publicaram sobre o tema são Nor Lela Ahmad e Wan Edura Wan Rashid, com nove publicações cada. Essas nove publicações, no entanto, são as mesmas, demonstrando uma parceria de autoria entre Ahmad e Rashid. Essa parceria estende-se também aos autores Nik Hasnaa Nik Mahmood e Noor Azlina Yunus, cujos trabalhos nessa temática são todos em conjunto com Ahmad e Rashid, caracterizando uma forte parceria de autoria.

\subsubsection{Países que mais publicam}

Em relação aos países, a Austrália destaca-se como aquele com maior número de publicações na área (80 publicações). Em seguida, destacam-se Estado Unidos e Reino Unido, havendo uma grande diferença em quantidade de publicações entres estes e o país seguinte, o Canadá (Figura 3).

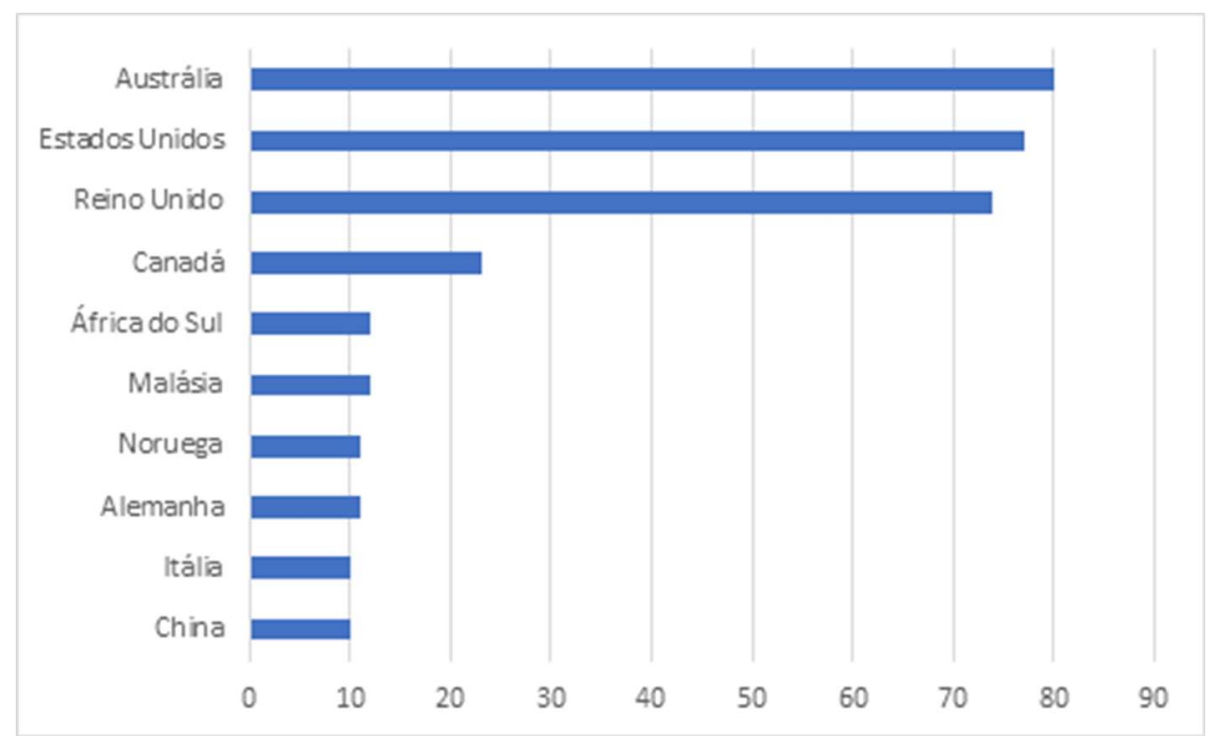

Figura 3. Países que mais publicam

Fonte: elaborado pelos autores (2019). 
Os dados mostram que há, portanto, uma concentração de publicações sobre a temática no eixo Austrália - Estados Unidos - Reino Unido. Essa concentração, no entanto, é constituída por uma configuração bastante pulverizada de autores, não ficando restrita a um grupo de autores específicos. Configuração contrária pode ser apontada no caso da Malásia. Quatro dos oito autores mais profícuos na área de gestão de eventos - Ahmad, Rashid, Mahmood e Yunus - são da Malásia (ou seja, fora do eixo Austrália - Estados Unidos - Reino Unido). A produção malasiana representa cerca de $4 \%$ da produção total englobada pela amostra desta pesquisa, o que representa 12 trabalhos. Desses, nove são de autoria dos referidos autores. Todos os quatro autores são filiados a Universiti Teknologi MARA.

Em relação ao Brasil, somente dois trabalhos brasileiros foram identificados na amostra, sendo um de Sousa-Mast, Reis, Gurgel e Duarte (2013), e outro de Piggin et al. (2019), ambos tratando dos Jogos Olímpicos de 2016 que aconteceram na cidade do Rio de Janeiro, RJ.

\subsubsection{Instituições que mais publicam}

Além da Universiti Teknologi MARA, destacam-se também (Figura 4) as instituições University of Queensland (a que mais publica) e Griffith University, ambas australianas; e a Leeds Beckett University, localizada no Reino Unido. Esses dados podem ser explicados pelo fato de que todas essas instituições possuem programas específicos de formação em gestão de eventos, com destaque para a University of Queensland e a Leeds Beckett University, que além de graduação, também oferecem curso na área com nível de pós-graduação.

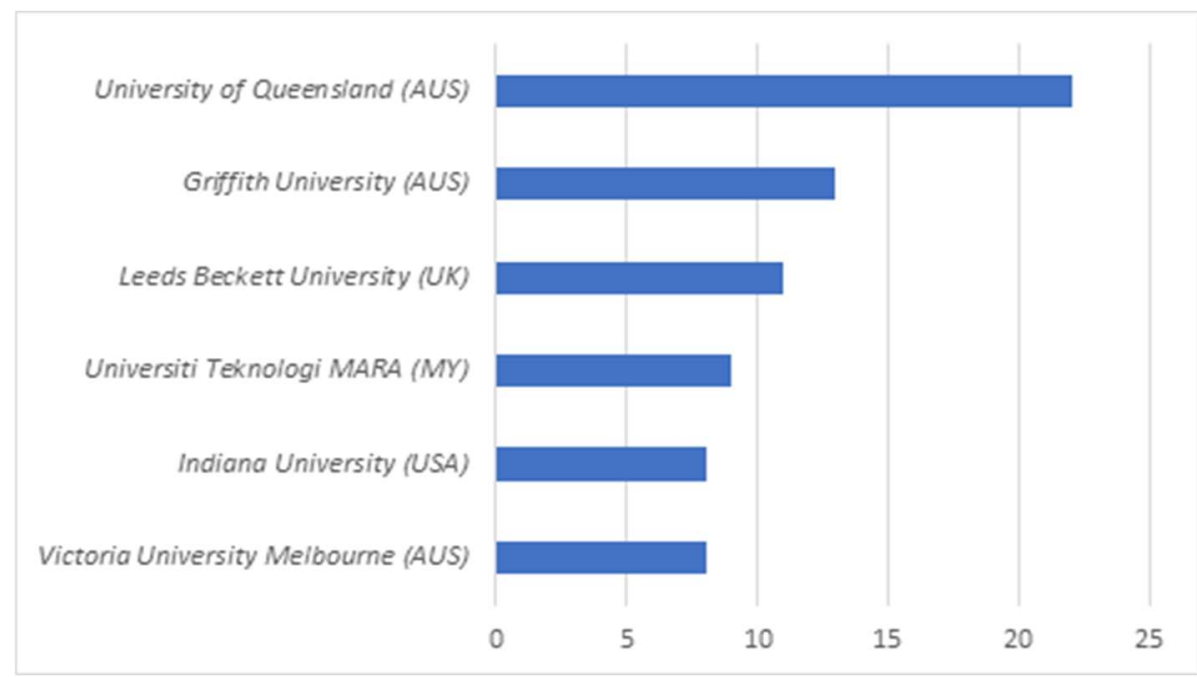

Figura 4. Instituições que mais publicam

Fonte: elaborado pelos autores (2019). 
Dessa forma, infere-se que existe um ambiente propício à produção científica sobre gestão de eventos nessas instituições, o que explica o destaque conferido a elas. Da mesma forma, isso ajuda a explicar o destaque do eixo Austrália - Estados Unidos - Reino Unido que ficou evidenciado na Figura 3 anteriormente.

\subsubsection{Composição de autoria}

A amostra total de trabalhos possui uma composição de autoria que varia entre trabalhos com apenas um autor até trabalhos que possuem oito autores (vide Figura 5). A maior parte deles (123 trabalhos) possui dois pesquisadores como autores.

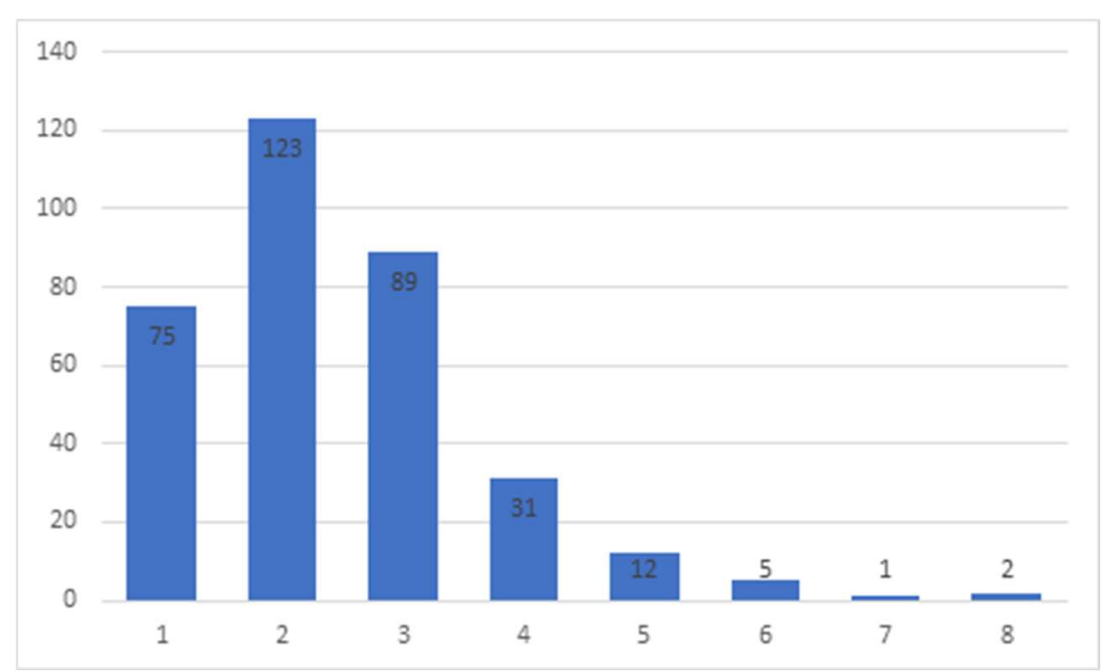

Figura 5. Composição de autoria

Fonte: elaborado pelos autores (2019).

Analisando os trabalhos com sete e oito autores, considerados outliers diante do cenário geral de composição de autoria, verificou-se que esses trabalhos versam sobre assuntos diversos à gestão, tais como ensino superior em gestão de eventos e promoção de saúde em megaeventos.

\subsection{Periódicos que mais publicam}

Os 338 artigos que compõem a amostra desta pesquisa estão publicados em 107 periódicos (uma média de 3,2 por periódico). A Tabela 1 mostra os periódicos com maior quantidade de publicações na área de gestão de eventos. Para fins de melhor visualização, a tabela divide-se em três zonas, cada uma com aproximadamente um terço do total de trabalhos publicados, segmentação proposta por Lima e Leocádio (2018). 
Periódicos com mais publicações

\begin{tabular}{|c|c|}
\hline Periódicos & Quantidade de artigos \\
\hline $1^{\mathrm{a}}$ zona -2 periódicos & 111 \\
\hline Event Management (USA) & 73 \\
\hline $\begin{array}{c}\text { International Journal of Event and Festival } \\
\text { Management }(U K)\end{array}$ & 38 \\
\hline $2^{\mathrm{a}}$ zona -15 periódicos & 113 \\
\hline $3^{\mathrm{a}}$ zona -90 periódicos & 114 \\
\hline
\end{tabular}

Fonte: elaborada pelos autores (2019).

Conforme mostra a Tabela 1, na primeira zona, apenas dois periódicos são responsáveis por 111 dos 338 artigos que compõem a amostra da pesquisa, sendo estes periódicos especializados no assunto. Na segunda zona, agora composta por 15 periódicos, encontram-se 113 publicações. Por fim, a última zona concentra 114 publicações e é composta por 90 periódicos, 45 vezes mais periódicos do que a primeira zona.

Dessa forma, confirma-se a lei bibliométrica de Bradford, conforme Rousseau e Rousseau (2000), segundo a qual muitos periódicos, com produtividade mais dispersa, publica a mesma quantidade de trabalhos que um pequeno grupo de periódicos que é mais produtivo em um dado tema. Permite-se, assim, estimar a alta relevância (Machado et al., 2016) que os periódicos Event Management e International Journal of Event and Festival Management possuem em suas áreas.

Ainda sobre a característica dessas publicações, a maioria se concentra na área de estudos de Administração e Contabilidade (290 publicações), dado este que não surpreende tendo em vista que se trata de um tema ligado à gestão. Chama a atenção, no entanto, a ocorrência de publicações na área de Ciências Ambientais/Energia (31 publicações), confirmando que a temática da sustentabilidade tem ganhado espaço na área de gestão de eventos, mostrando-se uma tendência de pesquisa.

\subsection{Publicações mais citadas}

Em relação às publicações mais citadas, levando-se em conta o critério de número de citações (corte: mais de 100 citações), obteve-se a Tabela 2: 
Tabela 2

Publicações mais citadas

\begin{tabular}{|c|c|c|c|}
\hline Autor(es) (Ano) & Título & Periódico & $\begin{array}{l}\mathrm{N}^{\circ} \text { de } \\
\text { citações }\end{array}$ \\
\hline Getz, D. (2008) & $\begin{array}{c}\text { Event tourism: Definition, evolution, and } \\
\text { research }\end{array}$ & Tourism Management & 742 \\
\hline $\begin{array}{l}\text { Green, B. C., Chalip, } \\
\text { L. (1998) }\end{array}$ & $\begin{array}{l}\text { Sport tourism as the celebration of } \\
\text { subculture }\end{array}$ & $\begin{array}{l}\text { Annals of Tourism } \\
\text { Research }\end{array}$ & 195 \\
\hline $\begin{array}{l}\text { Farrell, J.M., } \\
\text { Johnston, M.E., } \\
\text { Twynam, G.D. (1998) }\end{array}$ & $\begin{array}{c}\text { Volunteer motivation, satisfaction, and } \\
\text { management at an elite sporting } \\
\text { competition }\end{array}$ & $\begin{array}{l}\text { Journal of Sport } \\
\text { Management }\end{array}$ & 181 \\
\hline Green, C. B. (2001) & $\begin{array}{c}\text { Leveraging Subculture and Identity to } \\
\text { Promote Sport Events }\end{array}$ & $\begin{array}{l}\text { Sport Management } \\
\text { Review }\end{array}$ & 142 \\
\hline $\begin{array}{l}\text { Lee, W., Xiong, L., } \\
\text { Hu, C. (2012) }\end{array}$ & $\begin{array}{c}\text { The effect of Facebook users' arousal and } \\
\text { valence on intention to go to the festival: } \\
\text { Applying an extension of the technology } \\
\text { acceptance model }\end{array}$ & $\begin{array}{c}\text { International Journal of } \\
\text { Hospitality } \\
\text { Management }\end{array}$ & 136 \\
\hline $\begin{array}{l}\text { Kim, H., Borges, } \\
\text { M.C., Chon, J. (2006) }\end{array}$ & $\begin{array}{c}\text { Impacts of environmental values on } \\
\text { tourism motivation: The case of FICA, } \\
\text { Brazil }\end{array}$ & Tourism Management & 125 \\
\hline
\end{tabular}

Fonte: elaborado pelos autores (2019).

Com 742 citações, o trabalho Event tourism: Definition, evolution, and research, de Getz (2008) (também um dos autores mais profícuos - vide Figura 2), é a obra mais citada no campo. Uma possível explicação reside no fato de Getz (2008) realizar uma revisão da literatura do campo de turismo de eventos, analisando o fenômeno do ponto de vista teórico e prático, oferecendo contribuições tanto acadêmicas, quanto profissionais, o que representou um relevante esforço de sistematização do campo.

Destaca-se também a autora Green, que possui dois dos seis trabalhos $(1998 ; 2001)$ mais citados apontados neste estudo. O primeiro, Sport tourism as the celebration of subculture, é datado de 1998, em parceria com Chalip; portanto, um dos primeiros trabalhos identificados na área de gestão de eventos. Esse pioneirismo pode ser apontado como uma das razões para o fato de o trabalho ser bastante citado, uma vez que trouxe implicações para o marketing e para as teorias do turismo. O segundo trabalho, Leveraging Subculture and Identity to Promote Sport Events, de 2001, trata do mesmo tema, consolidando a contribuição da autora para o campo do comportamento do consumidor e do marketing aplicado à gestão de eventos e ao turismo esportivo.

Os trabalhos apresentados na Tabela 2, portanto os mais citados, tratam de temáticas diversas entre si (turismo, eventos esportivos, comportamento do consumidor, intenção do 
público, impacto ambiental), corroborando com a caracterização dada por Silvers et al. (2006), Getz (2007), Zanella (2011) e Park e Park (2017), cujos trabalhos concordam sobre o aspecto de diversidade dos eventos e das temáticas de pesquisa a eles relacionados.

Ressalta-se que um dos estudos apresentados na Tabela 2 foi realizado no Brasil (Kim, Borges, \& Chon, 2006), analisando as diferenças motivacionais dos participantes de um evento turístico ecológico realizado no estado de Goiás. O estudo já tinha sido citado 125 vezes até a realização desta pesquisa.

Ainda, dos autores apontados pela Figura 2 como os mais profícuos na temática, somente Getz aparece entre aqueles mais citados, o que mostra que nem sempre o autor que mais publica em determinado assunto é necessariamente o mais citado em seu campo de estudo.

\subsection{Rede de coocorrência de palavras-chave}

A relação de coocorrência entre palavras-chave busca determinar quando tais termos ocorrem conjuntamente em uma determinada amostra, apareçam elas no título, no resumo ou na lista de palavras-chave (Eck \& Waltman, 2014). Dessa forma, é possível caracterizar temáticas, tendências e lacunas de pesquisas em uma determinada área. Quanto maior o nó, maior a frequência do termo; quanto mais próximos os nós, mais forte a relação entre eles.

Para a Figura 6, a rede foi formada a partir de palavras-chave com o mínimo de 5 ocorrências na amostra, resultando em uma configuração de 34 termos, organizados em nove clusters. Para facilitar a visualização, a Tabela 3 mostra as palavras-chave que tiveram até 10 ocorrências, ou seja, os nós mais representativos da rede. 


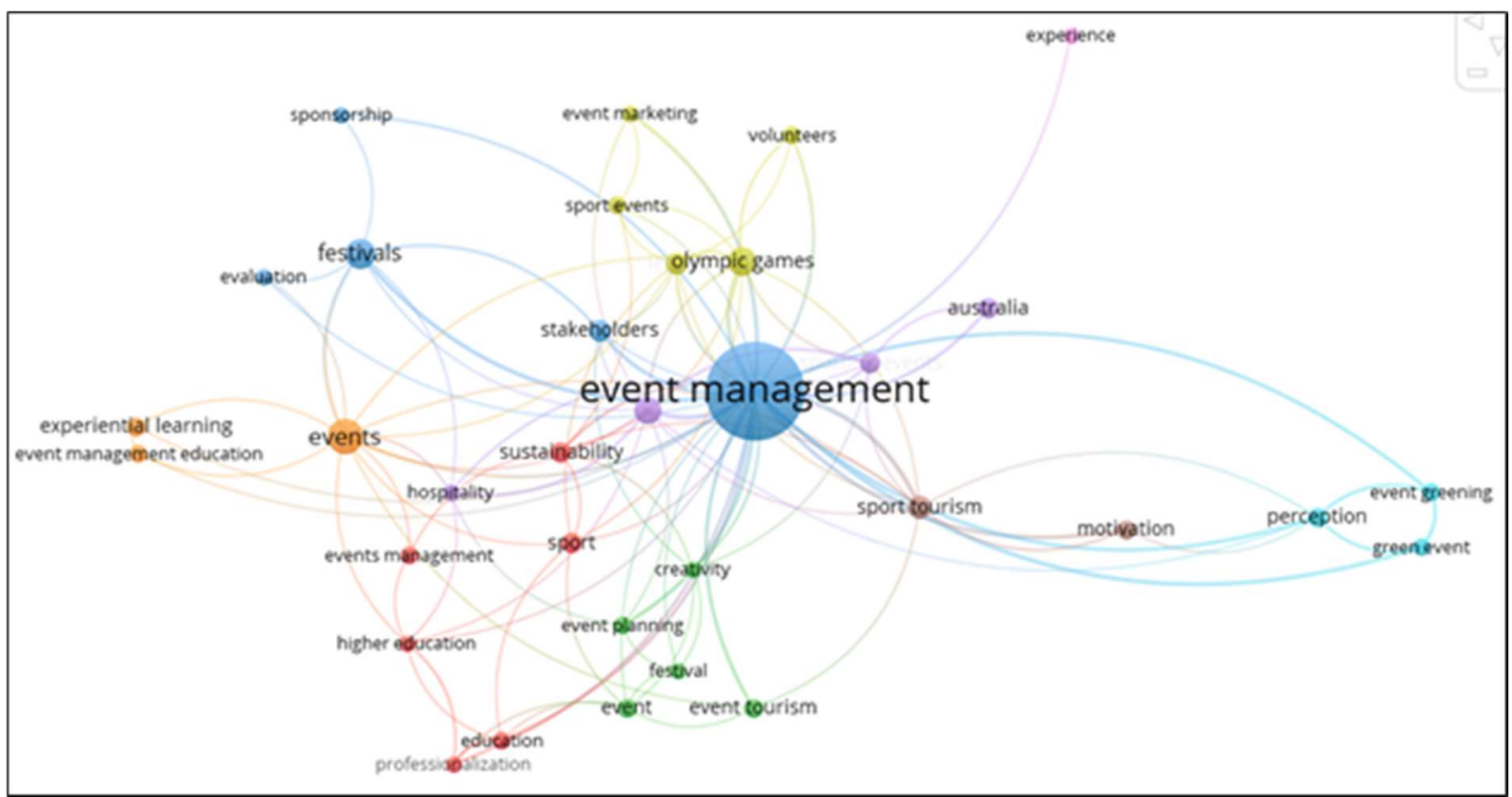

Figura 6. Rede de coocorrência de palavras-chave

Fonte: Elaborada pelos autores (2019).

Tabela 3

Palavras-chave com mais ocorrências

\begin{tabular}{cc}
\hline Palavra-chave & $\mathbf{N}^{\mathbf{0}}$ de ocorrências \\
\hline Event management & 129 \\
Events & 21 \\
Festivals & 16 \\
Olympic games & 14 \\
Tourism & 13 \\
Sport tourism & 10
\end{tabular}

Fonte: Elaborada pelos autores (2019).

Como esperado, o termo principal é event management, uma vez que se trata do próprio termo de busca que originou a amostra desta pesquisa. $\mathrm{O}$ termo aparece acoplado no cluster 3 , junto às palavras-chave avaliação, festivais, patrocínio e stakeholders, indicando um grupo de trabalhos que trata de gestão de eventos e festivais relacionada aos atores envolvidos em seu processo (patrocinadores e colaboradores/interessados). Esse resultado evidencia o que Mair e Whitford (2013) identificaram em seu estudo, que concluiu que uma das principais temáticas trabalhadas pelas produções científicas se relacionava justamente às políticas de governança envoltas na gestão de eventos, o que diz respeito ao relacionamento com stakeholders e patrocinadores. 
O cluster com mais palavras-chave é o cluster 1, cujos termos que o compõem são: educação, gestão de eventos (plural), educação superior, esporte e sustentabilidade. Tal grupo representa estudos ligados à formação e à profissão do gestor de eventos, enfatizando os achados de Park e Park (2017), para quem a formação/educação para gestão de eventos constituía uma temática recorrente nas pesquisas da área.

Tais achados consolidam as contribuições de Silvers et al. (2006), cujo estudo reconhece a gestão de eventos como um campo profissional que demanda habilidades e formações próprias, o que passa por um processo de educação específico e focado em eventos.

O cluster 1 abarca ainda estudos focados em eventos esportivos e na sustentabilidade. Em adição a esse dado, o cluster 6 traz os termos green event (evento verde) e event greening (que pode ser traduzido como "esverdeamento" de eventos, referindo-se ao processo de tornar os eventos mais sustentáveis). Entretanto, esse cluster não apresenta ligações com a palavrachave sustentabilidade presente no cluster 1, o que indica que aqueles termos compõem um arcabouço próprio e distinto deste último. Tal distância é corroborada, inclusive, pelo posicionamento do cluster 6 na rede, ao extremo direito da rede, encontrando-se mais afastado do centro e do nó do termo sustentabilidade.

Ainda, destacam-se as palavras-chave criatividade e turismo de eventos (cluster 2); marketing de eventos, legado e jogos olímpicos (cluster 4); e Austrália e hospitalidade (cluster 5), indicando um grupo concentrado de trabalhos que tratam de turismo, marketing e legado, corroborando os achados de Park e Park (2017) e Mair e Whitford (2013).

Assim, de acordo com a lei de Zipf, essas são as palavras-chave que, por sua frequência de ocorrência, determinam as temáticas centrais da produção científica em gestão de eventos (Chang, 2016; Lima \& Leocádio, 2018).

Prosseguindo com a análise de coocorrência de palavras-chave, buscou-se verificar os termos que se destacam considerando o ano de publicação dos artigos, a fim de encontrar, dessa forma, tendências de pesquisa da área em relação ao tempo. Assim, mantendo os critérios da rede apresentada na Figura 6, obteve-se a Figura 7. 


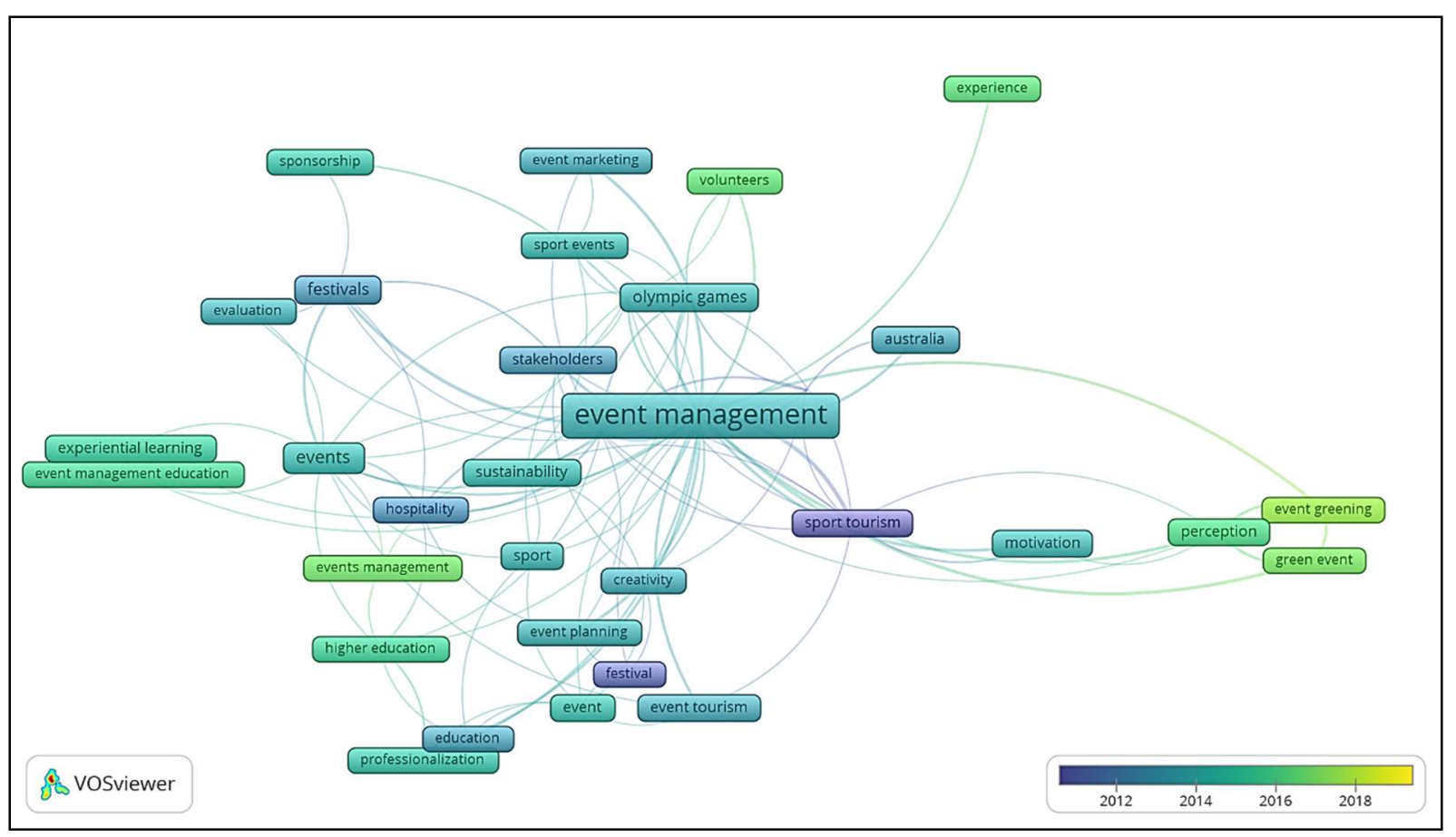

Figura 7. Palavras-chave versus ano

Fonte: Elaborada pelos autores (2019).

A primeira observação da rede apresentada na Figura 7 recai sobre o fato de que somente aparecem palavras-chave de artigos publicados a partir de 2011. Isso significa que os artigos de anos precedentes não atenderem ao critério mínimo de 5 ocorrências para serem incluídas na rede.

A segunda observação é que termos como eventos verdes e "esverdeamento" de eventos são recentes entre os artigos da área de gestão de eventos, indicando uma tendência de pesquisa no campo, o que parece atender ao que preconizavam Mair e Whitford (2013) sobre a necessidade de estudos sobre os impactos ambientais da realização de eventos.

Palavras-chave como educação superior e educação em gestão de eventos também podem ser consideradas tendências recentes, apresentando-se evidentes em meados de 2015. Tal resultado corrobora com aquele apontado por Park e Park (2017), que também enfatizavam a temática de formação/educação como uma das mais pesquisadas em tempos recentes na área de gestão de eventos.

\subsection{Rede de coautoria}

Para a análise de coautoria da amostra desta pesquisa, foram selecionados autores com um mínimo de 3 documentos e força total de ligação de valor mínimo 1. Essa restrição resultou em uma rede com 23 nós (autores), distribuídos em 8 clusters, conforme Figura 8. 


\section{fairley s.}

arcodia c.

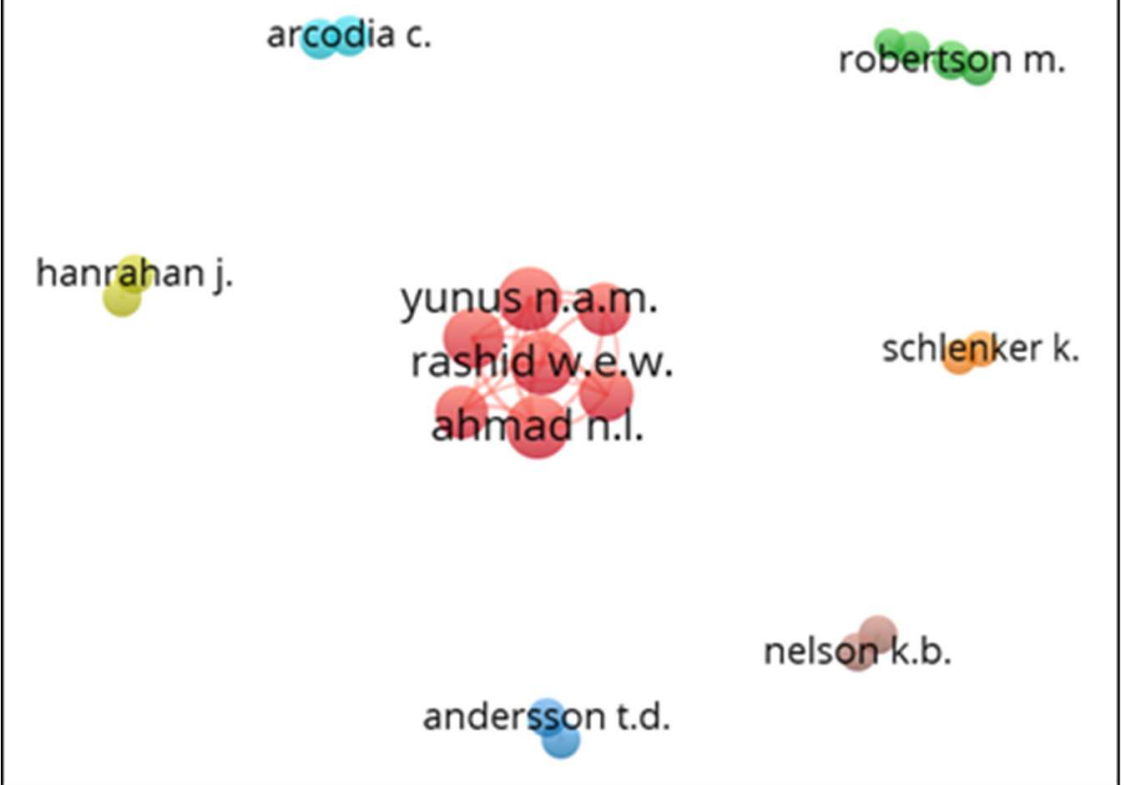

Figura 8. Rede de coautoria

Fonte: elaborada pelos autores (2019).

Os demais 360 autores ficaram de fora da rede em razão da restrição aplicada. Esse dado confirma a dispersão da pesquisa no campo de gestão de eventos, sinalizando uma imensa produção composta por autores que publicaram apenas dois ou menos artigos e que não apresentam nenhuma ligação.

Os clusters com mais nós, ou seja, com mais autores colaborando entre si são o cluster 1 e 2. O cluster 1 é formado por sete autores, sendo eles Ahmad, N.L., Mahmood, N. H. N., Ong, M. H. A., Rashid, W.E.W, Shamsuddin, S., Yunus, N.A.M. e Zamzuri, N.H. A análise de rede de coautoria deste cluster corrobora a forte parceria entre estes autores, conforme já havia sido identificado pela análise de autores mais profícuos (Figura 2). Em uma análise mais profunda, percebeu-se ainda que todas as publicações de Rashid e Yunus são em parceria com Ahmad e/ou Mahmood, corroborando a força de ligação do cluster. Os autores são todos vinculados a Universiti Teknologi MARA.

O cluster 2 é formado por quatro autores, sendo eles Ali-Knight, J., Barron, P., Lockstone-Binney, L. e Robertson, M. Como características do grupo, esses autores são vinculados a instituições localizadas na Austrália e no Reino Unido e a coautoria entres eles iniciou-se em 2008, sendo uma colaboração de pouco mais de uma década. Os demais clusters são formados por no máximo 2 autores, apresentando-se distantes entre si. 
Assim, observando a relação autores - instituições - países, de acordo com o que preconiza Rosa et al. (2010) e Eck e Waltman (2014), verifica-se a existência de uma maioria de clusters compostos por poucos autores que, conforme a estrutura de rede, estão distantes uns dos outros, corroborando para a característica dispersa da produção nesse campo.

\subsection{Rede de cocitação}

Neste estudo, optou-se analisar a cocitação da amostra pesquisada a partir da unidade de análise autores, por permitir conclusões mais objetivas. A Figura 9 mostra como se configura a rede de cocitação da amostra estudada.

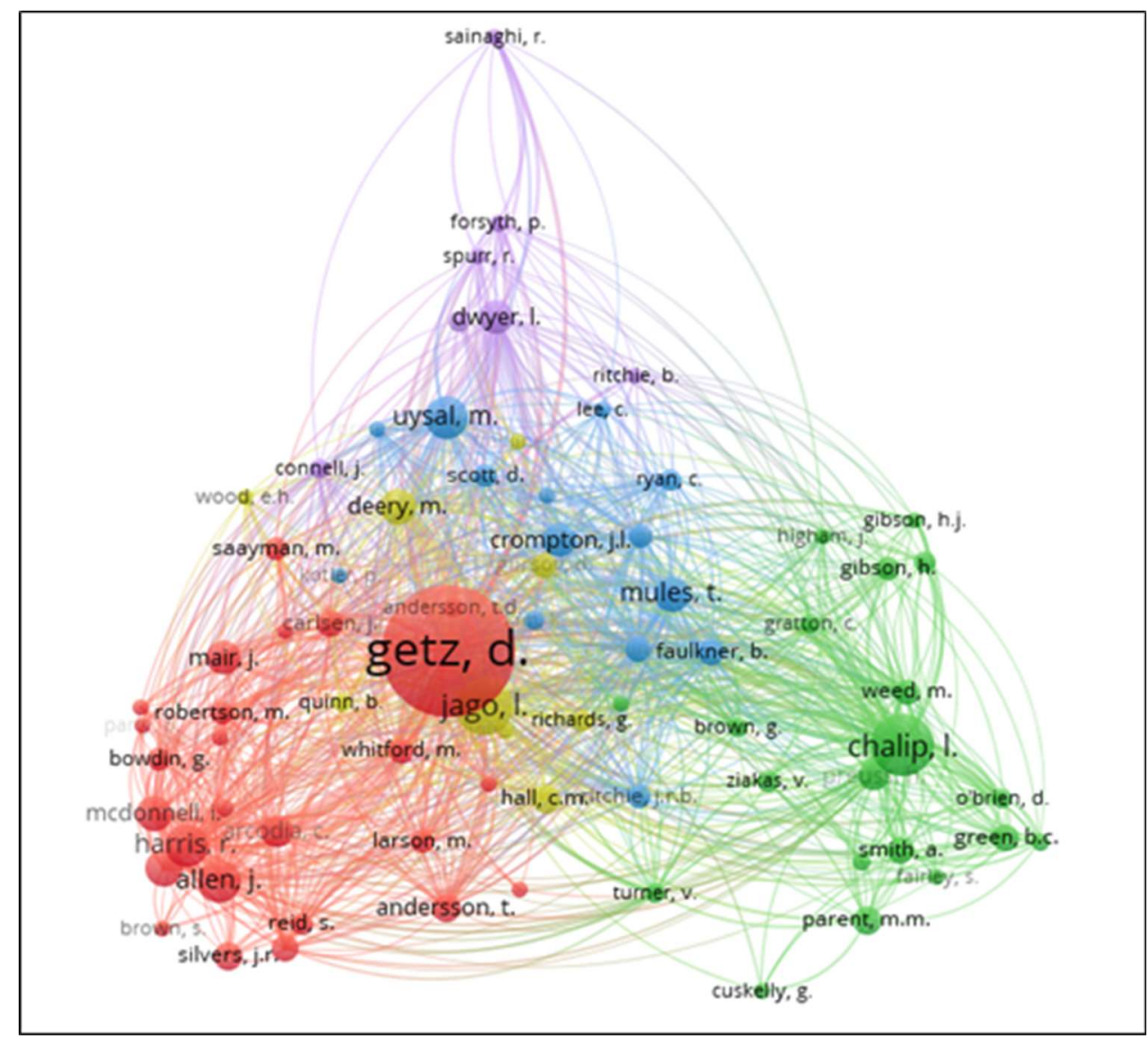

Figura 9. Rede de cocitação

Fonte: elaborada pelos autores (2019).

Como critério, estabeleceu-se o mínimo de 25 citações, o que resultou em uma rede com 76 autores, distribuídos em 5 clusters. Na figura, quanto maior o nó, maior a quantidade de citações que o autor recebeu; assim como quanto mais próximos os nós, mais forte é a relação entre eles.

O primeiro cluster é formado por 26 autores, destacando-se Getz, D (529 citações), Allen, J. (104 citações), Harris, R. (104 citações), O’Tolle, W. (84 citações) e McDonnell, I (79 
citações). Analisando seus trabalhos, observa-se que este cluster abriga autores que publicam principalmente sobre sustentabilidade, turismo, desenvolvimento comunitário e cidades.

O segundo cluster é formado por 20 autores, destacando-se Chalip, L. (180 citações), Preuss, H. (65 citações) e Parent, M. M. (56 citações). Analisando seus trabalhos, observa-se que este cluster abriga autores que publicam principalmente sobre eventos esportivos de grande porte, legado desses eventos, governança e relação com stakeholders.

O terceiro cluster possui 14 autores, sendo Uysal, M. o autor mais citado, com 104 citações. Ao seu lado, Mules, T. (82 citações) e Crompton, J. L. (74 citações) compõem os mais citados do cluster, que publica principalmente sobre eventos esportivos e análise de motivação e satisfação.

No quarto cluster, o destaque fica com Jago, L. (156 citações), Deery, M. (78 citações) e Hall, C. M. (56 citações), que tratam de temáticas eventos esportivos, gestão de recursos humanos voltada para gestão de eventos, stakeholders, legado e sustentabilidade.

Por fim, o quinto cluster tem como principal autor Dwyer, L. (71 citações). Todos os demais foram citados menos de 50 vezes. Considerando os trabalhos desse cluster de maneira geral, verifica-se que também tratam de temáticas como eventos esportivos, legados e benefícios, governança, stakeholders e voluntários.

Assim, conforme Cardoso et al., 2015), os cinco clusters representados na figura apontam para cinco agrupamentos de conteúdo. Dessa forma, parece haver uma predominância de temáticas ligadas a eventos esportivos e relações com stakeholders. No entanto, chama a atenção o fato de que o cluster com mais citações esteja relacionado a temáticas como sustentabilidade e impacto de eventos nas cidades e nas comunidades, denotando uma frente de pesquisa associada à gestão de eventos, o que parece responder à demanda de pesquisas sobre o tema levantada por Mair e Whtiford (2003).

\subsection{Rede de acoplamento bibliográfico}

Para análise desta rede, utilizou-se "autores" como unidade de análise, considerando-se apenas aqueles que possuíam pelo menos 3 artigos na amostra. Essa restrição resultou em uma rede com 34 autores, distribuídos em quatro clusters, conforme Figura 10. 


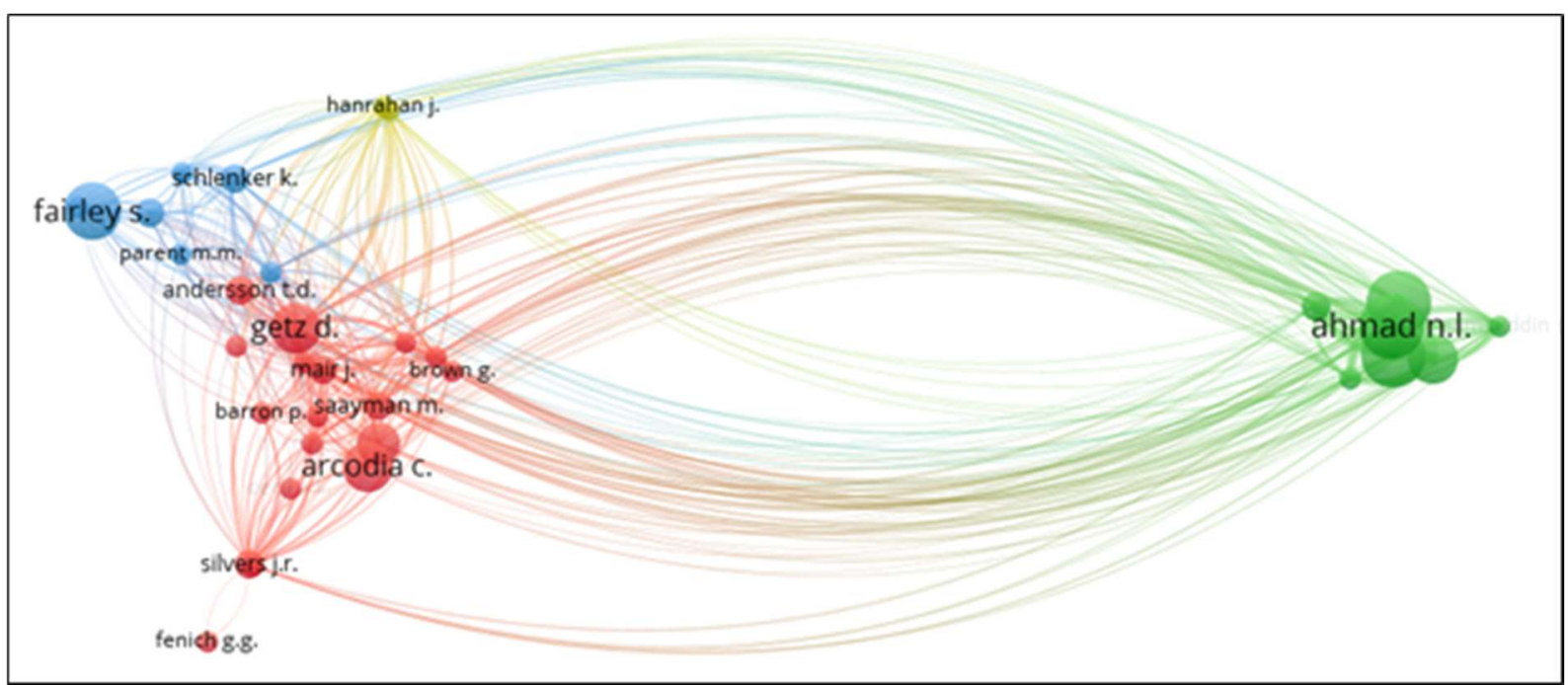

Figura 10. Rede de acoplamento bibliográfico

Fonte: Elaborada pelos autores (2019).

O primeiro cluster é formado por 18 autores, dentre os quais Getz D., Arcodia C., Andersson T. D e Silvers J. R. Esse cluster caracteriza-se por concentrar autores do eixo Austrália - Estados Unidos - Reino Unido, com predominância de instituições australianas.

O cluster 2, em contrapartida, caracteriza-se por ser mais isolado, encontrando-se no ponto extremo direito da rede. Como se pode observar, o grupo está distante dos demais clusters, indicando pouco acoplamento bibliográfico com estes. Esse grupo é formado por sete autores, os mesmos que também constituem um cluster próprio na rede de co-autoria: Ahmad, N. L.; Mahmood, N. H. N.; Ong, M. H. A.; Rashid, W. E. W.; Shamsuddin, S.; Yunus, N. A. M.; e Zamzuri, N. H. Esses autores são todos vinculados a Universiti Teknologi MARA, na Malásia, o que pode explicar parcialmente o isolamento identificado como característico do grupo.

Os clusters 3 e 4, por fim, são formados por sete e dois autores, respectivamente, e são mais acoplados bibliograficamente ao cluster 1, o que, conforme Lima e Leocádio (2018), indica núcleos referenciais de pesquisas em comum entre estes clusters.

\section{Considerações finais}

Esta pesquisa teve o objetivo de apresentar as características da produção científica internacional sobre gestão de eventos. Para tal, analisou uma amostra de 338 artigos científicos disponibilizados na base Scopus, realizando análises bibliométricas e sociométricas.

Os resultados permitem concluir que a pesquisa na área de gestão de eventos encontrase em ascensão, concentrando-se principalmente no eixo Austrália - Estados Unidos - Reino 
Unido. Uma característica comum entre esses países é que eles apresentam cursos de formação de nível superior específicos para o campo de gestão de eventos (em instituições que também são as mais profícuas no campo), além de contarem com periódicos especializados. Fora desse eixo, destaca-se um cluster de autores malasianos que, apesar de serem os mais profícuos na área, constituem um grupo isolado dos demais, com acoplamento bibliográfico forte entre si, mas fraco em relação aos demais clusters.

As leis bibliométricas de Lotka e Bradford foram confirmadas na amostra pesquisada, demonstrando que, na área de gestão de eventos, um pequeno grupo de autores e um pequeno grupo de periódicos são responsáveis por concentrar muitos publicações do tema. Em relação aos autores, destacam-se Nor Lela Ahmad e Wan Edura Wan Rashid; em relação aos periódicos, destacam-se o Event Management (EUA) e o International Journal of Event And Festival Management (Reino Unido). No entanto, os autores mais profícuos não são aqueles cujas obras são as mais citadas na área. Neste caso, destacam-se os autores Getz (2008), com o trabalho Event tourism: Definition, evolution, and research; e Green (1998), com as obras Sport tourism as the celebration of subculture, em co-autoria com Chalip, L.

Quanto à lei de Zipf, estudos sobre educação e profissionalização em gestão de eventos; turismo e eventos esportivos; marketing; e sustentabilidade e eventos verdes parecem constituir temas centrais e tendências na pesquisa em gestão de eventos. Esses achados, a partir da análise de coocorrência de palavras-chave, também corroboram para a conclusão de que a área se caracteriza como sendo bastante diversa.

Assim, identificou-se uma predominância de estudos sobre turismo de evento, eventos esportivos e festivais, bem como identificou-se uma lacuna de produção brasileira em bases internacionais. Essa escassez de trabalhos brasileiros também se repete em bases nacionais, informando-nos sobre a necessidade e, principalmente, sobre a oportunidade de pesquisa científica na área, sugerindo um campo amplo a ser explorado por aqueles que desejam se debruçar sobre a temática.

Como limites, esta pesquisa concentrou-se apenas em publicações internacionais em língua inglesa alocadas na base Scopus, o que restringe o universo amostral. Novas pesquisas podem ser feitas ampliando a base de busca e acessando publicações em outros idiomas.

\section{Referências}

Alvarenga, L. (1998). Bibliometria e arqueologia do saber de Michel Foucalt: traços de identidade teórico-metodológica. Ciência da Informação, 27(3), 1-9. 
Baas, J., Schotten, M., Plume, A., Côté, G., \& Karimi, R. (2020). Scopus as a curated, highquality bibliometric data source for academic research in quantitative science studies. Quantitative Science Studies, 1(1), 377-386.

Baum, T., Lockstone-Binney, L. \& Robertson, M. (2013). Event studies: finding fool's gold at the rainbow's end? International Journal of Event and Festival Management, 4(3), 179185.

Bowdin, G., Allen, J., O’Toole, W., Harris, R. \& McDonnell, I. (2011). Events Management (3rd ed.), Oxford: Butterworth-Heinemann.

Cardoso, A. L. J., Martins, T. S., \& Kato, H. T. (2015). Tendências temáticas em capacidades dinâmicas: um mapeamento do campo por meio de um estudo de cocitação. RAI-Revista de Administração e Inovação, 12(2), 38-59.

Chang, Y. (2016). Influence of human behavior and the principle of least effort on library and information science research. Information Processing \& Management, 52(4), 658-669.

Durán-Sánchez, A., Río-Rama, M. C. \& Álvarez-García, J. Bibliometric analysis of publications on wine tourism in the databases Scopus and WoS. European Research on Management and Business Economics, 23(1), 8-15.

Eck, N. J., van, \& Waltman, L. (2014). Visualizing bibliometric networks. In Y. Ding, R. Rousseau, \& D. Wolfram. (Eds.). Measuring scholarly impact: methods and practice (pp. 285-320). London: Springer.

Farrell, J.M., Johnston, M.E. \& Twynam, G.D. (1998). Volunteer motivation, satisfaction, and management at an elite sporting competition. Journal of Sport Management, 12(4), 288300 .

Getz, D. (2007). Event studies: theory, research and policy for planned events (1st ed.), Oxford: Butterworth-Heinemann.

Getz, D. (2008). Event tourism: Definition, evolution, and research. Tourism Management, 29(3), 403-428.

Getz, D. (2012). Event studies: discourses and future directions. Event Management, 16(2), 171-187.

Green, B. C., \& Chalip, L. (1998). Sport tourism as the celebration of subculture. Annals of Tourism Research, 25(2), 275-291.

Green, B. C. (2001). Leveraging Subculture and Identity to Promote Sport Events. Sport Management Review, 4(1), 1-19.

Kim, H., Borges, M. C. \& Chon, J. (2006). Impacts of environmental values on tourism motivation: The case of FICA, Brazil. Tourism Management, 27(5), 957-967. 
Lee, W., Xiong, L. \& Hu, C. (2012). The effect of Facebook users' arousal and valence on intention to go to the festival: applying an extension of the technology acceptance model. International Journal of Hospitality Management, 31(3), 819-827.

Lima, S. H. O. \& Leocádio, A. L. (2018). Mapeando a produção internacional sobre inovação aberta. Revista Brasileira de Gestão e Inovação, 5(2), 181-208.

Lotka, A. J. (1926). The frequency distribution of scientific productivity. Journal of the Washington Academy of Sciences, 16(12), 317-323.

Machado, C., Jr., Souza, M. T. S., Parisotto, I. R. S. \& Palmisano, A. (2016). As Leis da Bibliometria em diferentes bases de dados científicos. Revista de Ciências da Administração, 18(44), 111-123.

Mair, J. \& Whitford, M. (2013). An exploration of events research: event topics, themes and emerging trends. International Journal of Event and Festival Management, 4(1), 6-30.

Moreno, J. L. (1992). Quem sobreviverá? Fundamentos da sociometria, psicoterapia de grupo e sociodrama. Goiânia: Dimensão Editora.

Park,S. B. \& Park, K. (2017). Thematic trends in event management research. International Journal of Contemporary Hospitality Management, 29(3), 848-861.

Piggin, J., Souza, D. L., Furtado, S., Milanez, M., Cunha, G., Louzada, B. H., Graeff, B. \& Tlili, H. (2019). Do the Olympic Games promote dietary health for spectators? An interdisciplinary study of health promotion through sport. European SSport Management Quarterly, 19(4), 481-501.

Rosa, A. F., Mendes, A. C. A., Teixeira, G. M. A. \& Martins, S. (2010). Earnings Management no Brasil: uma análise sob a perspectiva sociométrica e bibliométrica. Revista Contabilidade Vista \& Revista, 21(4), 189-218.

Rousseau, B. \& Rousseau, R. (2000). Percolation as a model for informetric distributions: fragment size distribution characterized by Bradford curves. Scientometrics, 47, 195-206.

Silvers, J. R., Bowdin, G. J., O’Toole, W., \& Nelson, K. B. (2006). Towards an international event management body of knowledge (EMBOK). Event Management, 9, 185-198.

Sousa, E. S., Fontenele, R. E. S., Silva, A. L. L. \& Sousa, J. M., Filho. (2019). Mapeamento da produção científica internacional sobre intenção empreendedora. Revista Gestão $e$ Secretariado (GeSec), 10(3), 114-139.

Sousa-Mast, F. R., Reis, A. C., Gurgel, L. A. \& Duarte, A. F. P. L. A. (2013). Are cariocas getting ready for the Games? Sport participation and the Rio de Janeiro 2016 Olympic Games. Managing Leisure, 18(4), 331-335. 
Teixeira, M. L. M., Iwamoto, H. M. \& Medeiros H. L. (2013). Estudos bibliométricos em administração: discutindo a transposição de finalidade. Administração: Ensino e Pesquisa, $14(3), 423-452$.

Wagen, L., van der, \& Carlos, B. R. (2005). Event management for tourism, cultural, business, and sporting events. New Jersey: Pearson.

Zago, A. A., Domingues, C. R. \& Silva, A. M. S. (2019). Mobilidade internacional profissional: uma revisão bibliométrica. Revista Gestão e Secretariado (GeSec), 10(1), p. 119-140.

Zanella, L. C. (2011). Manual de Organização de Eventos: planejamento e operacionalização (4a ed.). São Paulo, SP: Atlas.

Submetido em: 06.10 .2020

Aceito em: $\quad 12.02 .2021$ 